\begin{tabular}{|l|l|}
\hline & $\begin{array}{l}\text { HHS PUblic ACCESS } \\
\text { Author manuscript } \\
\text { Microbiol Spectr. Author manuscript; available in PMC } 2017 \text { March } 01 .\end{array}$ \\
\hline
\end{tabular}

Published in final edited form as:

Microbiol Spectr. 2016 December ; 4(6): . doi:10.1128/microbiolspec.TNMI7-0023-2016.

\title{
Diabetes and tuberculosis
}

Blanca I. Restrepo

UTHealth Houston, School of Public Health at Brownsville, 80 Fort Brown, Brownsville, Texas 78520, blanca.i.restrepo@uth.tmc.edu or blancares2008@gmail.com, Telephone: (956)

279-3841; Fax: (956) 882-5152

\section{Keywords}

tuberculosis; diabetes; immunity; epidemiology; clinical; public health

\section{INTRODUCTION}

The global increase in type 2 diabetes mellitus (DM) is a recognized re-emerging risk and challenge to tuberculosis (TB) control (1). Individuals with DM have three times the risk of developing TB and there are now more individuals with TB-DM co-morbidity than TB-HIV co-infection. $(2,3)$ The association between DM and TB was first described by centuries ago by Avincenna, a persian philosopher, and the co-morbidity was a frequent topic in the medical literature from the first half of the XXth century.(4-7) But this literature dwindled as the association reduced notiriety with the introduction of insulin for diabetes patients and antibiotics for TB. In the 1980s the publications on joint TB-DM began to re-emerge in parallel with the DM 'pandemic': The global prevalence of DM among adults has increased by $20 \%$ in less than 30 years (8), and DM is predicted to reach 642 million worldwide by 2040 with most (80\%) of the patients living in low and middle-income countries where TB is also endemic. (9) Consequently, the World Health Organization has identified DM as a neglected, important and re-emerging risk factor for TB (1). In this chapter 'DM' will refer mostly to type $2 \mathrm{DM}$ since it is the most prevalent form, but type $1 \mathrm{DM}$ in children has also been associated with TB. $(9,10)$ This chapter describes the epidemiology of TB-DM, the impact of DM on the clinical presentation and outcomes of TB, the underlying biology that favors the co-occurrence of both diseases, and the public health implications for TB control and DM management.

\section{EPIDEMIOLOGY OF TB-DM}

\section{DM as a risk factor for TB}

Diabetes prevalence has increased worldwide as a result of population ageing, urbanisation, changes in diet and reduced physical activity patterns resulting in increasing obesity (11). About $80 \%$ of the 415 million estimated DM cases globally are from low and middle income countries and the DM prevalence is projected to rise most steeply in regions with high TB incidence over the next 30 years (9). A systematic review of 13 observational studies found that DM increases the risk of TB by three-fold (relative risk 3.11; 95\% CI 2.27-4.26).(3) Even though this is the best-characterized aspect of the association between TB and DM, these findings present wide variation between studies with risk ratios ranging between 
0.99-7.83. This illustrates the complexity of studying DM as a risk factor for TB given the heterogeneity in DM populations worldwide with respect to their age, access to healthcare, level of glucose control, and the type and number of DM complications and medications. Furthermore, co-ocurrence of DM with other host characteristics can further synergize TB risk among DM patients, as suggested for DM plus smoking, micro and macro-vascular complications of DM, and even their social environment (12-14) This emphasizes the need for studies reporting a thorough characterization of DM and other host factors with multivariable analysis in order to reach reliable conclusions.

The prevalence of TB-DM is higher in low- and middle-income countries where TB and DM are most prevalent. Of the ten countries with the highest number of DM patients worldwide, 6 are classified as "high burden" for TB by the World Health Organization, meaning they contribute to $80 \%$ of the TB cases worldwide (Figure 1). As studies on the epidemiology of TB-DM increase worldwide, certain regions display particularly high prevalence rates of DM among TB, including South India (54\%), the Pacific Islands (40\%) and northeastern Mexico (36\%).(15-18) However, developed countries are not excempt, and can have subpopulations with similar hotspots, as is the case of US communities adjacent to Mexico where the DM prevalence among TB patients is nearly $40 \%$.(18) The co-ocurrence of TBDM is not likely to diminish anytime soon. Longitudinal analysis of 163 countries reported increased TB incidence in settings where DM prevalence increased over time (19). In the Mexican state of Tamaulipas, across the border with the US, TB-DM increased by at least 2.8\% among TB patients diagnosed between 2006-2013, and this was not explained by higher glucose testing implemented at TB clinics in Mexico (20)(Figure 2). A more remarkable increase of 83\% was reported for all Mexican states between 2000-2012. (21)

\section{Contribution of DM to TB control}

At the population level, the contribution of DM to TB is generally between 10-20\%, but can vary substantially, even within a country. For example, in the UK the general population attributable risk is $10 \%$, but rises to $20 \%$ in Asian males.(22) In countries where TB and DM are endemic such as India or Mexico, the population attributable risk reaches at least $20 \%$. $(23,24)$ In the Texas-Mexico border our findings are even more striking with data suggesting that DM is the underlying attributable risk for nearly one-third (28\%) of the adult TB cases, and 51\% among TB patients who are 35-60 years old. In this region, HIV contributes to only 3-6\% of the adult TB cases (18). Therefore, even though DM confers a significantly lower risk of TB at the individual level (3-fold) when compared to HIV (>20fold), in communities like these where the sheer number of DM patients is high, the contribution of DM to TB can be higher than HIV (25). A study using dynamic TB transmission models to analyze the potential effects of DM on TB epidemiology in 13 countries with high burden of TB concluded that stopping the rise of DM would avoid 6 million (95\% CI 5.1, 6.9) incident cases and 1.1 millin $(1.0,1.3)$ TB deaths in these countries in 20 years (26). Thus, every community worldwide needs to evaluate the prevalence of DM and its contribution to TB. This information is variable between regions and critical to guide for the most efficient use of limited resources for TB control programs. 


\section{Profile of the TB-DM patient}

The profile of TB-DM patients versus TB only is strikingly different, with TB-DM patients being older, obese and more likely to be females who are not likely to present behaviors classically associated with TB such as alcohol abuse, consumption of illicit drugs, incarceration or HIV-AIDS. Thus, physicians need to be trained in contemporary times to "think TB" when examining patients with pulmonary infections and a "non-classical" sociodemographic profile for TB, in order to avoid delays in TB diagnosis. TB-DM patients (versus TB only) are also more likely to have lower education and higher unemployment, which complicates TB and DM management given that these sociodemograhic factors are associated with less access to healthcare and poorer glucose control (20).

\section{Directionality of the association}

Most studies on TB-DM are observational with few cohorts (all retrospective based on medical records) so stict inference of directionality in the association is not possible. However, most data provides support for DM preceding TB. All cohorts to date indicate that DM develops before TB, and those with further characterization of the DM patients suggest that it is not DM in itself, but rather, poorly controlled DM that increases TB risk $(14,27$, 28) Cross-sectional studies also support the concept of DM preceding TB, with chronic DM patients (median 7 years) that present other DM complications prior to TB development (18, 20,29 ). This highlights the missed opportunities for preventing TB among DM patients who have presumably been in contact for years with their healthcare providers. However, TB-DM patients should be distinguished from TB patients with transient hyperglycemia secondary to the inflammation induced during TB.(30) Thus, a new DM diagnosis can only be established once the TB patient is no longer febrile.

\section{Impact of the association between TB and DM on early DM diagnosis and management}

Given that an estimated 50\% of DM patients in developing countries are not aware of their DM diagnosis, TB clinics are becoming hubs for new diagnosis of DM worldwide. Some studies show that newly-diagnosed DM patients with TB (versus previously-diagnosed DM) have a different profile: they are more likely to be males, younger patients, and with lower HbA1cs $(15,18,20)$. This highlights the importance of TB clinics to reach males who are not as likely to be in contact with the healthcare system when compared to females, or to identify patients who are at an earlier stage of their DM before the presentation of additional and irreversible micro- and macro-vascular complications of DM. Thus, a growing public health challenge for low- and middle-income countries where both diseases are most likely to converge, is to coordinate the long-term care required for DM with the immediate shortterm care required for TB control.

\section{CLINICAL PRESENTATION OF TB IN TB-DM PATIENTS AND PUBLIC HEALTH IMPLICATIONS}

Many studies suggest that DM is associated with the clinical presentation of TB. Namely, TB-DM patients (versus TB-no DM) are more likely to present with pulmonary (versus extra-pulmonary), cavitary (versus non-cavitary) and sputum smear-positive TB at diagnosis. 
During the course of TB treatment, TB-DM patients take longer to convert from sputum smear-positive to -negative. Some studies also find that DM patients are more likely to present with drug-resistant and multi-drug resistant TB, although this relationship is not seen in all studies (Figure 3). I expand on each of these:

\section{Pulmonary versus extra-pulmonary TB}

Pulmonary TB accounts for $70-80 \%$ of the cases, and it is generally accepted that immune compromise facilitates hematogenous dissemination of $\mathrm{Mtb}$, predisposing to extrapulmonary TB. Such is the case of TB patients with HIV-AIDS (31) or those taking TNF blockers (32). This contrasts with TB-DM patients who are less likely to present with extrapulmonary TB $(17,28,33,34)$. This may be due to a hyper-reactive cell-mediated immune response to Mtb in DM patients that may be suboptimal for containing Mtb growth within the lung, but effective for preventing its dissemination and reactivation elsewhere (35-37).

\section{Cavitary and smear-positive TB}

Mtb induces a strong cell-mediated immunity leading to the formation of pulmonary granulomas (tubercles) that are thought to be a double-edged sword for the host (38). Granulomas initially limit Mtb growth, but in hosts in whom Mtb continues to replicate, these structures undergo central caseation with rupturing and spilling of thousands of viable bacilli into the airways. This "cavitary TB" is associated with sputum smear-positivity (39). TB-DM patients are more likely than TB-no DM to present with cavitary TB that is accompanied by higher bacillary burden in sputa $(34,40)$.

Together, the higher frequency of PTB vs extrapulmonary TB, cavitary TB, and smearpositive TB at diagnosis and extending during treatment, would predict that TB-DM patients are more infectious than TB-no DM (41). Such studies have never been conducted, but if confirmed, this would mark another public health implication for the TB-DM co-morbidity.

\section{Drug and multi-drug resistant (MDR) TB}

The relationship between drug or MDR TB in DM is unclear, with conflicting findings on the association between higher drug or MDR-TB in TB-DM patients versus TB-no DM (20, 42-50) In a meta-analysis of publications up to 2010, the prevalence of drug-resistant or MDR TB among recurrent TB cases was not significantly higher in TB-DM patients (OR $1.24,95 \%$ CI $0.72,2.16)(51)$. However, these findings were based on only four studies. Hence, there is a need for more studies that systematically evaluate the relationship between MDR TB and DM co-morbidity, with appropriate testing for MDR TB among the entire population at the time of TB diagnosis (not just those with treatment failures), multivariable analysis to sort out the independent contribution of DM versus other confounders, and characteristics of the study population and protocols of the local TB control programs in order to understand the circumstances under which MDR TB and DM may synergize. 


\section{TB TREATMENT OUTCOMES IN TB-DM PATIENTS}

There is growing evidence from observational studies that TB-DM is associated with an increase in adverse TB treatment outcomes, specifically for delays in mycobacterial clearance, treatment failures, death, relapse and re-infection (Figure 3)(27, 52, 53).

\section{Delays in sputum smear clearance and treatment failure}

TB-DM versus TB-no DM patients are more likely to remain sputum smear-positive after completion of the intensive phase of treatment, and this outcome is an early predictor of treatment failure (sputum smear or culture positivity at five months or later during treatment), which is also more likely in TB-DM versus TB-no DM $(51,53,54)$.

\section{Death}

Death was a hallmark of the co-morbidity in the 1950s with studies reporting that patients with DM were likely to die from a diabetic comma or TB (4-7). In a systematic review and meta-analysis of contemporary literature, Baker et al concluded that the risk of death from TB or any other cause in 23 unadjusted studies was nearly 2-fold (RR 1.89; 95\% CI 1.52-2.36), and this increased to 4.95 (95\% CI 2.69-9-10) in 4 studies that adjusted for age and potential confounders (51).

\section{Relapse and re-infection}

TB-DM patients also appear to have a higher risk of relapse. The review by Baker et al reported a nearly 4-fold risk of relapse in TB-DM versus TB-no DM (RR 3.89; 95\% CI 2.43-6.23) (51). A prospective study in southern Mexico with 1262 TB patients characterized for Mtb genotypes further distinguished between relapses and re-infections, and found higher adjusted odds of both outcomes in DM vs no DM $(\mathrm{OR}=1.8$ for both recurrence and relapse) (53).

\section{Should TB-DM patients be managed differently from TB-no DM?}

The clinical findings and higher risk of adverse outcomes in TB-DM patients indicate the need for prospective cohort studies aimed at confirming these observations and identifying the underlying factors leading to treatment failures in DM. Two underlying factors are prime suspects. The first is poor glucose control. Chronic hyperglycemia is associated with the dysfunctional immunity to Mtb in DM patients (Figures 5), and hence, likely to reduce the efficiency of anti-mycobacterial treatment. Hyperglycemia may also compromise Mtb killing by affecting the microvasculature and reducing lung tissue perfusion for optimal immune surveillance. However, the need for intervention studies to assess the effect of glucose control on TB treatment outcomes is questionable (55), and the WHO considers that the available data is sufficient to recommend optimized glucose control as part of the management of TB-DM patients for improved TB outcomes (56). The second suspect is possible suboptimal plasma levels of anti-mycobacterial antibiotics in the DM versus nonDM patients (57-59). While these studies have conflicting results, further assessment of suboptimal drug levels and its relationship to treatment failure among TB-DM patients is required, particularly in the continuation phase of treatment (60). This may not only lead to treatment failure, but can favor the development of MDR-TB as discussed above. With the 
available information, a joint group of expert clinicians have provided recent guidelines for treatment of drug-susceptible TB with specific recommendations for DM patients. First, depending on the resources and epidemiology of the community, screening for DM should be performed on all new TB patients with age $>45$ years, body mass index $>25$, first-degree relative with diabetes and race/ethnicity of African American, Asian, Hispanic, American Indian/Alaska Native or Hawaiian Native/Pacific Islander. Second, pyridoxine (vitamin B6) should be given with INH to DM patients, given their higher risk of neuropathy. Third, given that DM patients are more likely to present cavitations, smear-positivity at diagnosis and/or remain culture-positive at 2 months of treatment, a continuation phase of seven months duration is recommended, for a total of 9 months of therapy. Fourth, consideration should be given to measuring their drug concentrations in serum (therapeutic drug monitoring) to gain insights into the adequacy of drug dosing and need for tailored adjustments. If the DM patient has end stage renal disease, then therapeutic drug monitoring may be necessary to adjust drug levels in the context of dialysis, assess interactions with other medications for their co-morbid condition, and monitor toxicity (61).

\section{BIOLOGICAL BASIS FOR THE ASSOCIATION BETWEEN TB AND DM}

Immunological impairment has played a major role in TB susceptibility throughout history, and with the DM pandemic, DM is now among the most common causes of compromised immunity that favor TB development in contemporary times, along with HIV/AIDS, malnutrition, aging and smoking $(62,63)$. But DM contrasts from these other underlying conditions in that the immunity against Mtb is not necessarily "compromised" but rather, "dysfunctional", with excessive and/or delayed responses against Mtb. A summary of the key innate and adaptive components of the immune response that affect TB outcomes is provided in Chapter 3.

Innate immunity to Mtb in the TB-naïve DM host-In studies to simulate the first encounter between Mtb and the innate immune response of a TB-naïve host with DM, we find that monocytes from healthy individuals with DM (versus non-DM) have significantly reduced association (binding and phagocytosis) of Mtb. This defect appears to be attributed to alterations in the diabetic monocyte itself as well as in serum opsonins for Mtb, particularly the $\mathrm{C} 3$ component of complement which mediates Mtb phagocytosis $(64,65)$ (Figure 4). These in-vitro findings in humans are consistent with in-vivo observations in mice with chronic DM where there is also a reduced uptake of Mtb by alveolar macrophages within two weeks of infection (66). Furthermore, this model of chronic DM in mice is associated with delayed innate immunity to Mtb due to late delivery of Mtb-bearing antigenpresenting cells to the lung draining lymph nodes (67). Efficient phagocytosis and priming of the adaptive immune responses are necessary to activate the cell-mediated immune response that restricts initial Mtb growth (67) and these delays likely contribute to the higher risk of DM patients for Mtb infection and persistence (Figure 3).

\section{Adaptive immune responses to Mtb in DM hosts with LTBI and TB \\ Studies in individuals with LTBI and DM-The scanty literature in individuals with LTBI and DM (LTBI-DM) versus LTBI-no DM, shows decreased frequencies of LTBI (68,}


69) It is possible that lower levels of pro-inflammatory cytokines in DM can favor progression from LTBI to TB in DM, but further studies are required to understand the impact of lower anti-inflammatory cytokines as well.

Studies in individuals with TB and DM-In contrast to LTBI, there are a number of immunological studies in DM patients with TB (TB-DM) that indicate a hyper-inflammatory response to Mtb antigens when compared to TB-no DM. Most studies indicate that the exvivo (whole blood) and in-vitro stimulation of peripheral white blood cells with mycobacterial antigens results in higher Th1 and Th17 responses, including higher IFN- $\gamma$ and IL-17 secretion (35-37) (Figure 5 for IFN- $\gamma$ ). Other cytokines promoting immunity have been also reported to be higher in TB-DM vs TB-no DM, such as IL-2 and GM-CSF (35, 37). TB-DM cases also have a higher frequency of single- and double-cytokine producing CD4+ Th1 cells in response to Mtb antigens (for IFN- $\gamma$, TNF-a or IL-2) (37). These hyperactive responses in peripheral blood contrast with the results from few studies conducted at the site of infection (in bronchoalveolar lavage) where TB-DM patients appear to have reduced activation of immunity; one reported a lower proportion of activated alveolar macrophages (70), and another higher anti-inflammatory (IL-10) and lower proinflammatory (IFN- $\gamma$ ) cytokines (71). The impact of the host compartment (peripheral blood versus lung) requires further study. In mice with chronic DM and TB, there is higher pulmonary Mtb burden and more extensive inflammation with higher expression of proinflammatory cytokines like IFN- $\gamma(67,72,73)$. These findings in the lungs of mice resemble the hyper-response to Mtb antigens in the peripheral blood of TB-DM (versus TBno DM) patients, with similar findings in the guinea pig model of TB-DM (74).

\section{Understanding the immune dysfunction to Mtb in DM patients-Given that} efficient Mtb killing by anti-mycobacterial antibiotics requires cooperation between innate and adaptive immune responses, the higher frequency of adverse outcomes in DM patients suggests that the hyper-reactive immune response to mycobacterial antigens in TB-DM patients is not effective for Mtb killing. There are several possible explanations for the contribution of dysfunctional immunity to these adverse treatment outcomes. 1) The higher Th1 and Th17 response is only present in the peripheral blood of TB-DM patients, while anti-inflammatory responses that facilitate Mtb growth only occur in the lungs. 2) There is a higher production of pro-inflammatory cytokines like IFN- $\gamma$ in the lungs of humans (as observed in mice), but it is not effective for downstream activation of macrophages or cytotoxic T-cells that ultimately kill Mtb. 3) The hyper-reaction to Mtb antigens may be deleterious and contribute to lung tissue damage with more severe TB and the higher frequency of death in TB-DM patients. Understanding this complex relationship between excessive immunity in TB-DM will help improve the clinical management of TB patients, regardless of their DM status. Current TB research interests include the identification of host-directed therapies that synergize with antibiotics for effective $M t b$ elimination. The goal of these host-directed therapies is to boost immune mechanisms that diminish excess inflammation to reduce lung tissue damage and limit $M t b$ growth. Coincidently, the most commonly-used medication for type 2 diabetes, metformin, is one of the candidates for TB host-directed therapy (75). Preliminary findings suggests metformin may be beneficial for TB control by reducing the deleterious inflammation associated with immune pathology and 
enhancing the anti-mycobacterial activity of immune cells (76). Additional studies are now required to further elucidate the underlying relationship between MetF and Mtb killing, with careful assessment of the risks involved by adding anti-inflammatory medications like MetF to the $\mathrm{TB}$ regimen (77).

\section{DM AND LTBI}

Risk of LTBI in patients with DM-The literature often refers to DM as a risk factor for reactivation of LTBI to TB, but the relative risk of primary versus reactivation TB has never been systematically studied in DM. That is, there is scanty information on whether DM increases the risk of successful LTBI in close contacts, and/or facilitates the progression from LTBI to TB. Indirect evidence suggests that both scenarios are possible. In a population-based study in TB patients from Southern Mexico, the proportion of Mtb strains with genotypes that were clustered (similar genotypes suggesting recent infection) versus non-clustered (different Mtb genotypes suggesting re-activation TB) did not differ between TB-DM and TB-no DM patients (Mtb genotype clusters in 24\% and non-clustered in $76 \%$ in both study groups)(24). However, other findings suggest that DM may increase the risk of primary TB. First, a cohort of close contacts of TB patients in China reported that DM was a risk factor for TB development within 5-years after the diagnosis of TB in the index case (adjusted OR 3.4, 95\%CI 1.0-11.3)(78). Second, TB-DM patients are more likely to have lower lobe lung infiltrates which is where Mtb implants upon inhalation. This is classical of primary $\mathrm{TB}$, when compared to upper lung infiltrates after lympho-hematogenous dissemination in reactivation TB (79). However, alternative explanations for the higher frequency of lower lung lobe infiltrates in DM are also possible (e.g. older age is more frequent in DM and also favors lower lung lobe infiltrates).

Immunoassays for diagnosis of LTBI (or TB): is there immune compromise in DM patients?-The in-vivo tuberculin skin test (TST) and ex-vivo Interferon-gamma release assays (IGRAs, namely QuantiFERON-TB Gold assays by Qiagen and T.Spot-TB by Oxford Immunotec) are used in the clinical setting to diagnose LTBI in individuals with high risk of TB progression (targeted testing), or to support the diagnosis of TB. These assays detect immunological memory to Mtb (or mycobacteria spp. for TST) and their performance depends on immune function. Given the contribution of these tools to TB prevention, there is a need to determine whether the immune dysfunction in DM compromises their performance. In TB patients +/- DM, most studies show that DM does not compromise the performance of TST and/or IGRAs, with some contradictory findings that may be attributed to lack of control for possible confounders (80-83).

There are few studies on the performance of the TST or IGRAs for the diagnosis of LTBI in DM patients. A community-based study in Tanzania showed that the proportion of QFT-IT negatives was higher among individuals with pre-DM or DM (vs no DM), suggesting reduced sensitivity of these assays in these patient population. However, this difference was not significant after controlling for confounders (81). Among refugees in Atlanta, US, there was a higher proportion of LTBI among those with pre-DM or DM (versus non-DM) when using QFT-IT (84) Thus, the few available studies do not indicate a clear compromise due to DM, but studies are needed to understand the individual contribution of DM and its synergy 
with additional host factors (older age, gender, BMI) that can collectively influence assay sensitivity.

\section{CONCLUDING REMARKS}

The converging pandemic of DM in low- and middle-income countries where TB is endemic has been identified as one of the factors that will hinder the global TB target of $90 \%$ reduction in TB incidence by 2035 (85). Several fronts need to be addressed: How can we prevent the development of TB among DM patients? How do we stratify among the millions of DM patients who have LTBI into those for higher TB risk for tailored and cost-effective recommendations for LTBI treatment? How can we reduce the prevalence of adverse TB treatment outcomes in TB-DM patients? A unifying factor from epidemiological, clinical and immunological studies on TB and DM is the importance of glucose control on TB risk and adverse TB treatment outcomes (55). Glucose control is also the mainstay for the prevention of other DM complications (86). Thus, it will be important to establish whether HbA1c in itself is a good predictor of TB risk among DM patients with LTBI, and how other biological or social factors can help refine risk estimates. But this apparently simple goal of achieving good glucose control among DM patients, particularly in low- and middle-income countries, is a major challenge that will require a multi-disciplinary approach.

Epidemiological, clinical and basic science research should guide the identification of simple and measurable risk factors for TB development at the individual level, and the public health and healthcare systems should make policy and adopt these findings. Only then will we be able to stratify TB risk with more precision for targeted TB prevention among the millions of DM patients at risk for TB.

\section{References}

1. Ottmani SE, Murray MB, Jeon CY, Baker MA, Kapur A, Lonnroth K, Harries AD. Consultation meeting on tuberculosis and diabetes mellitus: meeting summary and recommendations. Int J Tuberc Lung Dis. 2010; 14:1513-1517. [PubMed: 21180207]

2. Ronacher K, Joosten SA, van Crevel R, Dockrell HM, Walzl G, Ottenhoff TH. Acquired immunodeficiencies and tuberculosis: focus on HIV/AIDS and diabetes mellitus. Immunol Rev. 2015; 264:121-137. [PubMed: 25703556]

3. Jeon CY, Murray MB. Diabetes mellitus increases the risk of active tuberculosis: a systematic review of 13 observational studies. PLoS Med. 2008; 5:1091-1101.

4. Morton, R. Phthisiologia, Or a Treatise of Consumptions. London, England: 1694.

5. Boucot KR, Dillon ES, Cooper DA, Meier P, Richardson R. Tuberculosis among diabetics: the Philadelphia survey. Am Rev Tuberc. 1952; 65:1-50.

6. Root H. The Association of Diabetes and Tuberculosis. New England Journal of Medicine. 1934; 210:1-13.

7. Silwer H, Oscarsson PN. Incidence and coincidence of diabetes mellitus and pulmonary tuberculosis in a Swedish county. Acta Med Scand Suppl. 1958; 335:1-48. [PubMed: 13544883]

8. Danaei G, Finucane MM, Lu Y, Singh GM, Cowan MJ, Paciorek CJ, Lin JK, Farzadfar F, Khang YH, Stevens GA, Rao M, Ali MK, Riley LM, Robinson CA, Ezzati M, Global Burden of Metabolic Risk Factors of Chronic Diseases Collaborating G. National, regional, and global trends in fasting plasma glucose and diabetes prevalence since 1980: systematic analysis of health examination surveys and epidemiological studies with 370 country-years and 2.7 million participants. Lancet. 2011; 378:31-40. [PubMed: 21705069]

9. International Diabetes Federation. IDF Diabetes Atlas. 7th edn. Brussels, Belgium: 2015. vol http:// www.idf.org/diabetesatlas [last accessed 6/09/2016] 
10. Webb EA, Hesseling AC, Schaaf HS, Gie RP, Lombard CJ, Spitaels A, Delport S, Marais BJ, Donald K, Hindmarsh P, Beyers N. High prevalence of Mycobacterium tuberculosis infection and disease in children and adolescents with type 1 diabetes mellitus. Int J Tuberc Lung Dis. 2009; 13:868-874. [PubMed: 19555537]

11. Hu F. Globalization of diabetes: the role of diet, lifestyle, and genes. Diabetes Care. 2011; 34:1249-1257. [PubMed: 21617109]

12. Reed GW, Choi H, Lee SY, Lee M, Kim Y, Park H, Lee J, Zhan X, Kang H, Hwang S, Carroll M, Cai Y, Cho SN, Barry CE III, Via LE, Kornfeld H. Impact of diabetes and smoking on mortality in tuberculosis. PLoS ONE. 2013; 8:e58044. [PubMed: 23469139]

13. Lonnroth K, Jaramillo E, Williams BG, Dye C, Raviglione M. Drivers of tuberculosis epidemics: the role of risk factors and social determinants. Soc Sci Med. 2009; 68:2240-2246. [PubMed: 19394122]

14. Kuo MC, Lin SH, Lin CH, Mao IC, Chang SJ, Hsieh MC. Type 2 diabetes: an independent risk factor for tuberculosis: a nationwide population-based study. PLoS One. 2013; 8:e78924.

[PubMed: 24236069]

15. Kornfeld H, West K, Kane K, Kumpatla S, Zacharias RR, Martinez-Balzano C, Li W, Viswanathan V. High Prevalence and Heterogeneity of Diabetes in Patients With TB in South India: A Report from the Effects of Diabetes on Tuberculosis Severity (EDOTS) Study. Chest. 2016; 149:15011508. [PubMed: 26973015]

16. Viney K, Brostrom R, Nasa J, Defang R, Kienene T. Diabetes and tuberculosis in the Pacific Islands region. Lancet Diabetes Endocrinol. 2014; 2:932. [PubMed: 25433427]

17. Restrepo BI, Fisher-Hoch SP, Crespo JG, Whitney E, Perez A, Smith B, McCormick JB. Type 2 diabetes and tuberculosis in a dynamic bi-national border population. Epidemiol Infect. 2007; 135:483-491. [PubMed: 16863600]

18. Restrepo BI, Camerlin A, Rahbar M, Restrepo M, Zarate I, Wing R, Mora-Guzman F, Crespo-Solis J, Briggs J, McCormick J, Fisher-Hoch S. Cross-sectional assessment reveals high diabetes prevalence among newly-diagnosed tuberculosis patients. Bull World Health Organ. 2011; 89:352359. [PubMed: 21556303]

19. Goldhaber-Fiebert JD, Jeon CY, Cohen T, Murray MB. Diabetes mellitus and tuberculosis in countries with high tuberculosis burdens: individual risks and social determinants. Int J Epidemiol. 2011; 40:417-428. [PubMed: 21252210]

20. Abdelbary BE, Garcia-Viveros M, Ramirez-Oropesa H, Rahbar MH, Restrepo BI. Tuberculosisdiabetes epidemiology in the border and non-border regions of Tamaulipas, Mexico. Tuberculosis (Edinb). 2016 doi:10.1016/j.tube.2016.09.024.

21. Delgado-Sanchez G, Garcia-Garcia L, Castellanos-Joya M, Cruz-Hervert P, Ferreyra-Reyes L, Ferreira-Guerrero E, Hernandez A, Ortega-Baeza VM, Montero-Campos R, Sulca JA, MartinezOlivares Mde L, Mongua-Rodriguez N, Baez-Saldana R, Gonzalez-Roldan JF, Lopez-Gatell H, Ponce-de-Leon A, Sifuentes-Osornio J, Jimenez-Corona ME. Association of Pulmonary Tuberculosis and Diabetes in Mexico: Analysis of the National Tuberculosis Registry 2000-2012. PLoS One. 2015; 10:e0129312. [PubMed: 26075393]

22. Walker C, Unwin N. Estimates of the impact of diabetes on the incidence of pulmonary tuberculosis in different ethnic groups in England. Thorax. 2010; 65:578-581. [PubMed: 20421330]

23. Stevenson CR, Forouhi NG, Roglic G, Williams BG, Lauer JA, Dye C, Unwin N. Diabetes and tuberculosis: the impact of the diabetes epidemic on tuberculosis incidence. BMC Public Health. 2007; 7:234. [PubMed: 17822539]

24. Ponce-De-Leon A, Garcia-Garcia Md Mde L, Garcia-Sancho MC, Gomez-Perez FJ, ValdespinoGomez JL, Olaiz-Fernandez G, Rojas R, Ferreyra-Reyes L, Cano-Arellano B, Bobadilla M, Small PM, Sifuentes-Osornio J. Tuberculosis and diabetes in southern Mexico. Diabetes Care. 2004; 27:1584-1590. [PubMed: 15220232]

25. Getahun H, Gunneberg C, Granich R, Nunn P. HIV infection-associated tuberculosis: the epidemiology and the response. Clin Infect Dis. 2010; 50(Suppl 3):S201-S207. [PubMed: 20397949] 
26. Pan SC, Ku CC, Kao D, Ezzati M, Fang CT, Lin HH. Effect of diabetes on tuberculosis control in 13 countries with high tuberculosis: a modelling study. Lancet Diabetes Endocrinol. 2015; 3:323330. [PubMed: 25754415]

27. Baker MA, Lin HH, Chang HY, Murray MB. The risk of tuberculosis disease among persons with diabetes mellitus: a prospective cohort study. Clin Infect Dis. 2012; 54:818-825. [PubMed: 22238171]

28. Leung CC, Lam TH, Chan WM, Yew WW, Ho KS, Leung GM, Law WS, Tam CM, Chan CK, Chang KC. Diabetic control and risk of tuberculosis: a cohort study. Am J Epidemiol. 2008; 167:1486-1494. [PubMed: 18400769]

29. Stevenson CR, Critchley JA, Forouhi NG, Roglic G, Williams BG, Dye C, Unwin NC. Diabetes and the risk of tuberculosis: a neglected threat to public health? Chronic Illn. 2007; 3:228-245. [PubMed: 18083679]

30. Boillat-Blanco N, Ramaiya KL, Mganga M, Minja LT, Bovet P, Schindler C, Von Eckardstein A, Gagneux S, Daubenberger C, Reither K, Probst-Hensch N. Transient Hyperglycemia in Patients With Tuberculosis in Tanzania: Implications for Diabetes Screening Algorithms. J Infect Dis. 2016; 213:1163-1172. [PubMed: 26609005]

31. Leeds IL, Magee MJ, Kurbatova EV, del RC, Blumberg HM, Leonard MK, Kraft CS. Site of extrapulmonary tuberculosis is associated with HIV infection. Clin Infect Dis. 2012; 55:75-81. [PubMed: 22423123]

32. Harris J, Keane J. How tumour necrosis factor blockers interfere with tuberculosis immunity. Clin Exp Immunol. 2010; 161:1-9. [PubMed: 20491796]

33. Reis-Santos B, Locatelli R, Horta BL, Faerstein E, Sanchez MN, Riley LW, Maciel EL. SocioDemographic and Clinical Differences in Subjects with Tuberculosis with and without Diabetes Mellitus in Brazil - A Multivariate Analysis. PLoS ONE. 2013; 8:e62604. [PubMed: 23638123]

34. Viswanathan V, Kumpatla S, Aravindalochanan V, Rajan R, Chinnasamy C, Srinivasan R, Selvam JM, Kapur A. Prevalence of diabetes and pre-diabetes and associated risk factors among tuberculosis patients in India. PLoS ONE. 2012; 7:e41367. [PubMed: 22848473]

35. Restrepo BI, Fisher-Hoch S, Pino P, Salinas A, Rahbar MH, Mora F, Cortes-Penfield N, McCormick J. Tuberculosis in poorly controlled type 2 diabetes: altered cytokine expression in peripheral white blood cells. Clin Infect Dis. 2008; 47:634-641. [PubMed: 18652554]

36. Walsh M, Camerlin A, Miles R, Pino P, Martinez P, Mora-Guzman F, Crespo-Solis J, Fisher-Hoch S, McCormick J, Restrepo BI. Sensitivity of Interferon-gamma release assays is not compromised in tuberculosis patients with diabetes. Int J Tuberc Lung Dis. 2010; 15:179-184.

37. Kumar NP, Sridhar R, Banurekha VV, Jawahar MS, Nutman TB, Babu S. Expansion of pathogenspecific T-helper 1 and T-helper 17 cells in pulmonary tuberculosis with coincident type 2 diabetes mellitus. J Infect Dis. 2013; 208:739-748. [PubMed: 23715661]

38. Guirado E, Schlesinger LS, Kaplan G. Macrophages in tuberculosis: friend or foe. Semin Immunopathol. 2013; 35:563-583. [PubMed: 23864058]

39. Russell DG. Who puts the tubercle in tuberculosis? Nat Rev Microbiol. 2007; 5:39-47. [PubMed: 17160001]

40. Restrepo BI. Convergence of the tuberculosis and diabetes epidemics: renewal of old acquaintances. Clin Infect Dis. 2007; 45:436-438. [PubMed: 17638190]

41. Behr MA, Warren SA, Salamon H, Hopewell PC, Ponce de LA, Daley CL, Small PM. Transmission of Mycobacterium tuberculosis from patients smear-negative for acid-fast bacilli. Lancet. 1999; 353:444-449. [PubMed: 9989714]

42. Perez-Navarro LM, Fuentes-Dominguez FJ, Zenteno-Cuevas R. Type 2 diabetes mellitus and its influence in the development of multidrug resistance tuberculosis in patients from southeastern Mexico. J Diabetes Complications. 2015; 29:77-82. [PubMed: 25303784]

43. Bashar M, Alcabes P, Rom WN, Condos R. Increased incidence of multidrug-resistant tuberculosis in diabetic patients on the Bellevue Chest Service, 1987 to 1997. Chest. 2001; 120:1514-1519. [PubMed: 11713128]

44. Wang PD, Lin RS. Drug-resistant tuberculosis in Taipei, 1996-1999. Am J Infect Control. 2001; 29:41-47. [PubMed: 11172317] 
45. Subhash HS, Ashwin I, Mukundan U, Danda D, John G, Cherian AM, Thomas K. Drug resistant tuberculosis in diabetes mellitus: a retrospective study from south India. Trop Doct. 2003; 33:154156. [PubMed: 12870601]

46. Hsu AH, Lee JJ, Chiang CY, Li YH, Chen LK, Lin CB. Diabetes is associated with drug-resistant tuberculosis in Eastern Taiwan. Int J Tuberc Lung Dis. 2013; 17:354-356. [PubMed: 23228433]

47. Magee MJ, Bloss E, Shin SS, Contreras C, Huaman HA, Ticona JC, Bayona J, Bonilla C, Yagui M, Jave O, Cegielski JP. Clinical characteristics, drug resistance, and treatment outcomes among tuberculosis patients with diabetes in Peru. Int J Infect Dis. 2013; 17:e404-412. [PubMed: 23434400]

48. Gomez-Gomez A, Magana-Aquino M, Lopez-Meza S, Aranda-Alvarez M, Diaz-Ornelas DE, Hernandez-Segura MG, Salazar-Lezama MA, Castellanos-Joya M, Noyola DE. Diabetes and Other Risk Factors for Multi-drug Resistant Tuberculosis in a Mexican Population with Pulmonary Tuberculosis: Case Control Study. Arch Med Res. 2015; 46:142-148. [PubMed: 25704633]

49. Fisher-Hoch SP, Whitney E, McCormick JB, Crespo G, Smith B, Rahbar MH, Restrepo BI. Type 2 diabetes and multidrug-resistant tuberculosis. Scand J Infect Dis. 2008; 40:888-893. [PubMed: 18728934]

50. Magee MJ, Kempker RR, Kipiani M, Gandhi NR, Darchia L, Tukvadze N, Howards PP, Narayan KM, Blumberg HM. Diabetes mellitus is associated with cavities, smear grade, and multidrugresistant tuberculosis in Georgia. Int J Tuberc Lung Dis. 2015; 19:685-692. [PubMed: 25946360]

51. Baker MA, Harries AD, Jeon CY, Hart JE, Kapur A, Lonnroth K, Ottmani SE, Goonesekera SD, Murray MB. The impact of diabetes on tuberculosis treatment outcomes: a systematic review. BMC Med. 2011; 9:81. [PubMed: 21722362]

52. Jeon CY, Murray MB, Baker MA. Managing tuberculosis in patients with diabetes mellitus: why we care and what we know. Expert Rev Anti Infect Ther. 2012; 10:863-868. [PubMed: 23030325]

53. Jimenez-Corona ME, Cruz-Hervert LP, Garcia-Garcia L, Ferreyra-Reyes L, Delgado-Sanchez G, Bobadilla-del-Valle M, Canizales-Quintero S, Ferreira-Guerrero E, Baez-Saldana R, TellezVazquez N, Montero-Campos R, Mongua-Rodriguez N, Martinez-Gamboa RA, Sifuentes-Osornio J, Ponce-De-Leon A. Association of diabetes and tuberculosis: impact on treatment and posttreatment outcomes. Thorax. 2013; 68:214-220. [PubMed: 23250998]

54. Viswanathan V, Vigneswari A, Selvan K, Satyavani K, Rajeswari R, Kapur A. Effect of diabetes on treatment outcome of smear-positive pulmonary tuberculosis--a report from South India. J Diabetes Complications. 2014; 28:162-165. [PubMed: 24461545]

55. Jorgensen ME, Faurholt-Jepsen D. Is there an effect of glucose lowering treatment on incidence and prognosis of tuberculosis? A systematic review. Curr Diab Rep. 2014; 14:505. [PubMed: 24806518]

56. WHO. Disease IUATL. Collaborative framework for care and control of tuberculosis and diabetes. 2011. 2011http://www.who.int/diabetes/publications/tb_diabetes2011/en/. Accessed

57. Requena-Mendez A, Davies G, Ardrey A, Jave O, Lopez-Romero SL, Ward SA, Moore DA. Pharmacokinetics of rifampin in Peruvian tuberculosis patients with and without comorbid diabetes or HIV. Antimicrob Agents Chemother. 2012; 56:2357-2363. [PubMed: 22330931]

58. Nijland HM, Ruslami R, Stalenhoef JE, Nelwan EJ, Alisjahbana B, Nelwan RH, van d V, Danusantoso H, Aarnoutse RE, van CR. Exposure to rifampicin is strongly reduced in patients with tuberculosis and type 2 diabetes. Clin Infect Dis. 2006; 43:848-854. [PubMed: 16941365]

59. Babalik A, Ulus IH, Bakirci N, Kuyucu T, Arpag H, Dagyildizi L, Capaner E. Plasma concentrations of isoniazid and rifampin are decreased in adult pulmonary tuberculosis patients with diabetes mellitus. Antimicrob Agents Chemother. 2013; 57:5740-5742. [PubMed: 23979746]

60. Ruslami R, Nijland HM, Adhiarta IG, Kariadi SH, Alisjahbana B, Aarnoutse RE, van CR. Pharmacokinetics of antituberculosis drugs in pulmonary tuberculosis patients with type 2 diabetes. Antimicrob Agents Chemother. 2010; 54:1068-1074. [PubMed: 20038625]

61. Nahid P, Dorman SE, Alipanah N, Barry PM, Brozek JL, Cattamanchi A, Chaisson LH, Chaisson RE, Daley CL, Grzemska M, Higashi JM, Ho CS, Hopewell PC, Keshavjee SA, Lienhardt C, Menzies R, Merrifield C, Narita M, O’Brien R, Peloquin CA, Raftery A, Saukkonen J, Schaaf HS, Sotgiu G, Starke JR, Migliori GB, Vernon A. Executive Summary: Official American Thoracic Society/Centers for Disease Control and Prevention/Infectious Diseases Society of America 
Clinical Practice Guidelines: Treatment of Drug-Susceptible Tuberculosis. Clin Infect Dis. 2016; 63:853-867. [PubMed: 27621353]

62. Fox GJ, Menzies D. Epidemiology of tuberculosis immunology. Adv Exp Med Biol. 2013; 783:132. [PubMed: 23468101]

63. Rieder HL. The dynamics of tuberculosis epidemiology. Indian J Tuberc. 2014; 61:19-29. [PubMed: 24640341]

64. Gomez DI, Twahirwa M, Schlesinger LS, Restrepo BI. Reduced Mycobacterium tuberculosis association with monocytes from diabetes patients that have poor glucose control. Tuberculosis. 2013; 93:192-197. [PubMed: 23131496]

65. Restrepo BI, Twahirwa M, Rahbar MH, Schlesinger LS. Phagocytosis via Complement or FcGamma Receptors Is Compromised in Monocytes from Type 2 Diabetes Patients with Chronic Hyperglycemia. PLoS ONE. 2014; 9:e92977. [PubMed: 24671137]

66. Martinez N, Kornfeld H. Diabetes and immunity to tuberculosis. Eur J Immunol. 2014; 44:617626. [PubMed: 24448841]

67. Vallerskog T, Martens GW, Kornfeld H. Diabetic mice display a delayed adaptive immune response to Mycobacterium tuberculosis. J Immunol. 2010; 184:6275-6282. [PubMed: 20421645]

68. Kumar NP, George PJ, Kumaran P, Dolla CK, Nutman TB, Babu S. Diminished systemic and antigen-specific type 1 , type 17 , and other proinflammatory cytokines in diabetic and prediabetic individuals with latent Mycobacterium tuberculosis infection. J Infect Dis. 2014; 210:1670-1678. [PubMed: 24907382]

69. Kumar NP, Moideen K, George PJ, Dolla C, Kumaran P, Babu S. Coincident diabetes mellitus modulates Th1-, Th2-, and Th17-cell responses in latent tuberculosis in an IL-10- and TGF-betadependent manner. Eur J Immunol. 2016; 46:390-399. [PubMed: 26518995]

70. Wang CH, Yu CT, Lin HC, Liu CY, Kuo HP. Hypodense alveolar macrophages in patients with diabetes mellitus and active pulmonary tuberculosis. Tuber Lung Dis. 1999; 79:235-242. [PubMed: 10692992]

71. Sun Q, Zhang Q, Xiao H, Cui H, Su B. Significance of the frequency of CD4+CD25+C. Respirology. 2012; 17:876-882. [PubMed: 22524702]

72. Martens GW, Arikan MC, Lee J, Ren F, Greiner D, Kornfeld H. Tuberculosis Susceptibility of Diabetic Mice. American Journal of Respiratory Cell and Molecular Biology. 2007; 37:518-524. [PubMed: 17585110]

73. Yamashiro S, Kawakami K, Uezu K, Kinjo T, Miyagi K, Nakamura K, Saito A. Lower expression of Th1-related cytokines and inducible nitric oxide synthase in mice with streptozotocin-induced diabetes mellitus infected with Mycobacterium tuberculosis. Clin Exp Immunol. 2005; 139:57-64. [PubMed: 15606614]

74. Podell BK, Ackart DF, Obregon-Henao A, Eck SP, Henao-Tamayo M, Richardson M, Orme IM, Ordway DJ, Basaraba RJ. Increased severity of tuberculosis in Guinea pigs with type 2 diabetes: a model of diabetes-tuberculosis comorbidity. Am J Pathol. 2014; 184:1104-1118. [PubMed: 24492198]

75. Zumla A, Rao M, Parida SK, Keshavjee S, Cassell G, Wallis R, Axelsson-Robertsson R, Doherty M, Andersson J, Maeurer M. Inflammation and tuberculosis: host-directed therapies. J Intern Med. 2015; 277:373-387. [PubMed: 24717092]

76. Singhal A, Jie L, Kumar P, Hong GS, Leow MK, Paleja B, Tsenova L, Kurepina N, Chen J, Zolezzi F, Kreiswirth B, Poidinger M, Chee C, Kaplan G, Wang YT, De LG. Metformin as adjunct antituberculosis therapy. Sci Transl Med. 2014; 6:263ra159.

77. Restrepo BI. Metformin: Candidate host-directed therapy for tuberculosis in diabetes and nondiabetes patients. Tuberculosis (Edinb). 2016 doi:10.1016/j.tube.2016.09.008.

78. Lee MS, Leung CC, Kam KM, Wong MY, Leung MC, Tam CM, Leung EC. Early and late tuberculosis risks among close contacts in Hong Kong. Int J Tuberc Lung Dis. 2008; 12:281-287. [PubMed: 18284833]

79. Perez-Guzman C, Torres-Cruz A, Villarreal-Velarde H, Vargas MH. Progressive age-related changes in pulmonary tuberculosis images and the effect of diabetes. Am J Respir Crit Care Med. 2000; 162:1738-1740. [PubMed: 11069805] 
80. Rawat J, Sindhwani G, Biswas D. Effect of age on presentation with diabetes: Comparison of nondiabetic patients with new smear-positive pulmonary tuberculosis patients. Lung India. 2011; 28:187-190. [PubMed: 21886953]

81. Faurholt-Jepsen D, Aabye MG, Jensen AV, Range N, PrayGod G, Jeremiah K, Changalucha J, Faurholt-Jepsen M, Jensen L, Jensen SM, Krarup H, Ravn P, Friis H, Andersen AB. Diabetes is associated with lower tuberculosis antigen-specific interferon gamma release in Tanzanian tuberculosis patients and non-tuberculosis controls. Scand J Infect Dis. 2014; 46:384-391. [PubMed: 24621055]

82. Syed Ahamed KB, Raman B, Thomas A, Perumal V, Raja A. Role of QuantiFERON-TB gold, interferon gamma inducible protein-10 and tuberculin skin test in active tuberculosis diagnosis. PLoS ONE. 2010; 5:e9051. [PubMed: 20140219]

83. Jung JY, Lim JE, Lee HJ, Kim YM, Cho SN, Kim SK, Chang J, Kang YA. Questionable role of interferon-gamma assays for smear-negative pulmonary TB in immunocompromised patients. J Infect. 2012; 64:188-196. [PubMed: 22120597]

84. Hensel RL, Kempker RR, Tapia J, Oladele A, Blumberg HM, Magee MJ. Increased risk of latent tuberculous infection among persons with pre-diabetes and diabetes mellitus. Int J Tuberc Lung Dis. 2016; 20:71-78. [PubMed: 26688531]

85. Odone A, Houben RM, White RG, Lonnroth K. The effect of diabetes and undernutrition trends on reaching 2035 global tuberculosis targets. Lancet Diabetes Endocrinol. 2014; 2:754-764. [PubMed: 25194888]

86. Anonymous, The Diabetes Control and Complications Trial Research Group. The effect of intensive treatment of diabetes on the development and progression of long-term complications in insulin-dependent diabetes mellitus. N Engl J Med. 1993; 329:977-986. [PubMed: 8366922]

87. Restrepo BI, Schlesinger LS. Impact of diabetes on the natural history of tuberculosis. Diabetes Res Clin Pract. 2014; 106:191-199. [PubMed: 25082309] 




Figure 1.

Convergence of countries with highest burden of TB and DM worldwide. Among the ten countries with the highest number of diabetes patients worldwide, six are also among the 22 high-burden countries that contribute $80 \%$ of the TB cases worldwide (40). 


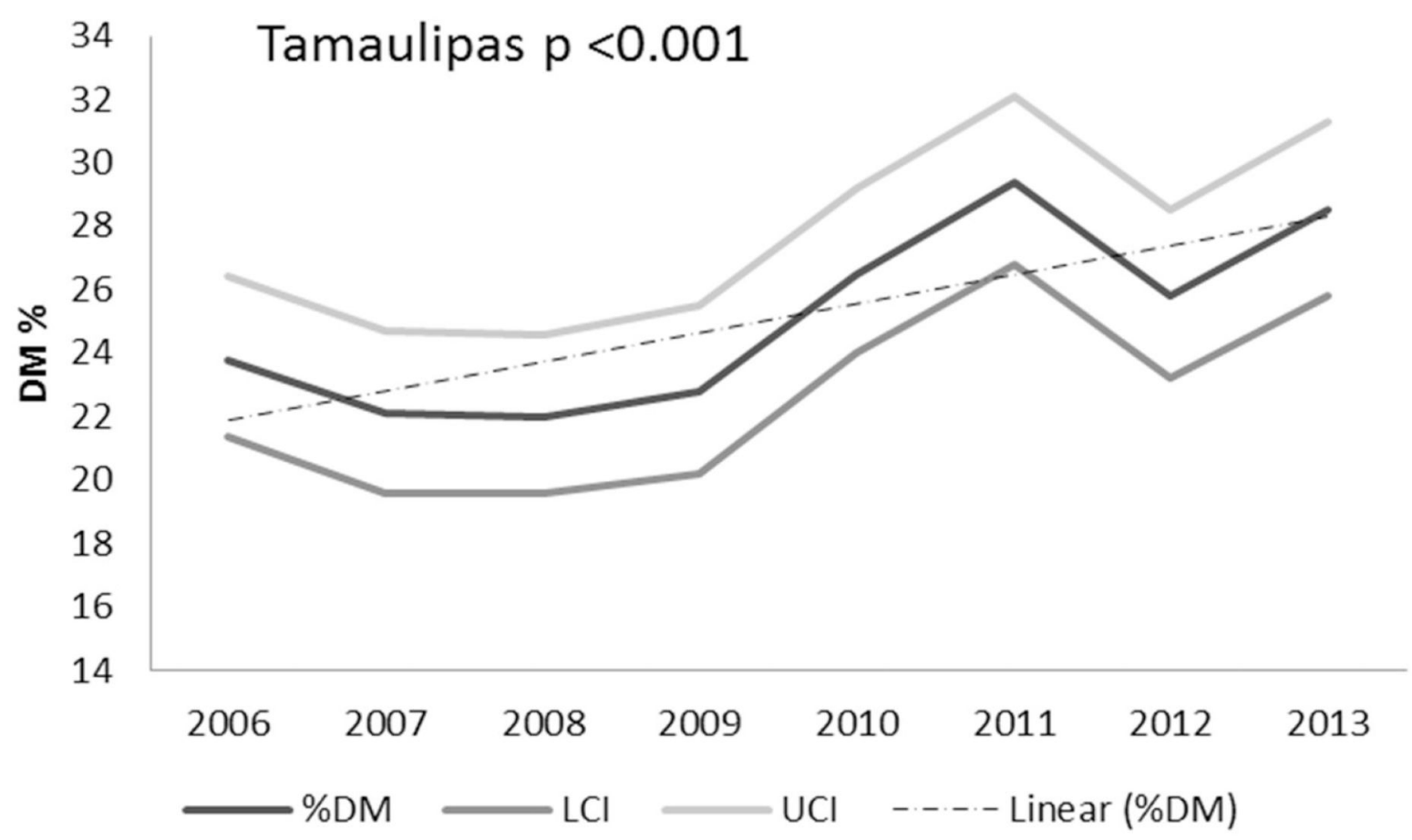

\section{Tamaulipas}

263
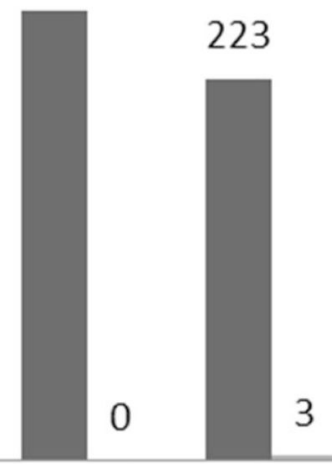

3

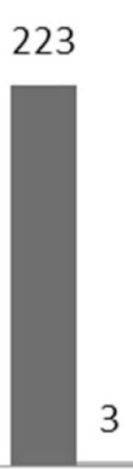

226

223
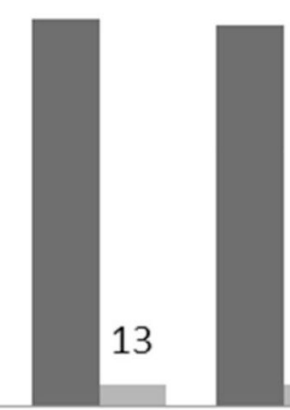

324

2006

$$
2007
$$

2008

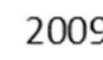

2010

2011

2012

口 DM\#

Figure 2.

Increase in the prevalence of TB-DM among TB cases over eight year period. Longitudinal analysis of the prevalence of TB-DM among newly-diagnosed TB patients reported to the state of Tamaulipas in northeastern Mexico, reveals an increase of 2.8\% between 2006 and 2014 that is not attributed to an increase in blood glucose testing for DM diagnosis at TB clinics. Adapted from reference (20), with permission. 




\begin{tabular}{|c|c|c|c|}
\hline IMMUNITY & $\begin{array}{ll} & \text { Mtb-naïve } \\
\text { DM (vs no DM): } \\
\text { - } \quad \text { Delayed T-cell } \\
\text { priming } \\
\text { - } \quad \downarrow \text { IFN- }- \\
\text { - } \quad \downarrow \text { Phagocytosis }\end{array}$ & $\begin{array}{c}\text { LTBI } \\
\text { DM (vs no DM): } \\
\cdot \quad \downarrow \text { Th1, Th17 } \\
\cdot \quad \downarrow \text { Th2 }\end{array}$ & \begin{tabular}{ll} 
& \multicolumn{1}{c}{ TB } \\
& DM (vs no DM): \\
- & $\uparrow$ Th1 and Th17 \\
- & 个 double cytokine- \\
& producing T cells \\
- & $\downarrow$ T-regulatory cells \\
- & 个IL-10 \\
- & $\frac{\text { Lungs: } \uparrow \text { IL-10 and }}{\downarrow I F N-\gamma}$
\end{tabular} \\
\hline
\end{tabular}

\section{CLINICAL PRESENTATION}

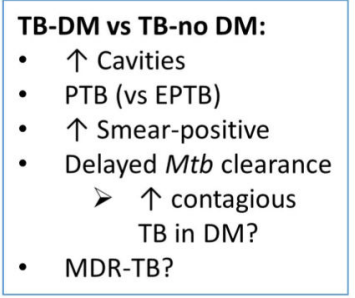

Figure 3.

Impact of diabetes on the natural history of TB: association with dysfunctional immunity and clinical characteristics. Exposure of Mtb-naïve individuals to a pulmonary TB patient generally results in no infection $(70 \%)$ or LTBI $(30 \%)$ or primary TB. Among those infected, the lifetime risk of reactivation TB is $10 \%$. If the host has DM (most likely chronic and poorly-controlled DM), the TB will increase by 3-fold, although the contribution of DM to LTBI, primary TB or re-activation TB has not been quantified. Once TB develops, possible treatment outcomes include cure, treatment failure or death. DM increases these outcomes. Among presumably cured individuals TB relapses can occur. A previous history of TB does not confer immunity against all strains and re-exposure to another Mtb strain can lead to re-infection. DM can also increase re-infection risks. Bold arrows and "DM" indicate stages of TB where DM appears to have an impact. As the natural history of TB evolves in the DM host, so does the immune response with characteristics that contrast with the nonDM host. The TB-DM host is more likely to present with clinical characteristics associated with enhanced TB transmission, but the impact of disease spread in the community has not been systematically studied (bottom text box; "DM?"). The dysfunctional immune response of the DM host to Mtb antigens is likely to influence the development, clinical presentation and outcomes of TB but the mechanisms involved are poorly understood (87). PTB, pulmonary TB; EPTB, extrapulmonary TB; LTBI, latent TB infection. 




Effect of serum heat-inactivation on M. tuberculosis association with monocytes from individuals $+/-D M$

\begin{tabular}{|c|c|c|c|c|}
\hline \multirow{3}{*}{$\begin{array}{c}D M \\
\text { classification }\end{array}$} & \multicolumn{4}{|c|}{$\%$ monocytes associated with $M$. tuberculosis } \\
\hline & \multicolumn{2}{|c|}{ Fresh serum $\left(A b+C^{\prime}\right)$} & \multicolumn{2}{|c|}{ Heated serum $(A b)$} \\
\hline & Mean +/- SD & $\mathrm{p}$ & Mean +/- SD & $p$ \\
\hline No DM & $28.4+/-8.0 \%$ & & $7.4+/-6.7 \%$ & \\
\hline$D M$ & $17.8+/-5.4 \%$ & ד. & $10.1+/-5.0 \%$ & ת.0 \\
\hline
\end{tabular}

Figure 4.

Lower phagocytosis of Mtb by monocytes from patients with DM. Monocytes from TBnaive individuals +/- DM were cultured in RPMI plus $20 \%$ fresh or heat-inactivated autologous serum. Mtb were added and after $2 \mathrm{~h}$ the non-bound bacteria were washed. Then the associated (bound or phagocytosed) Mtb were stained with auramine (orange) and monocyte nuclei with DAPI (blue)(left panel). The percent of monocytes with at least one associated Mtb was quantified using fluorescence microscopy. Adherence of Mtb to monocytes is medicated by antibodies to mycobacteria spp $(\mathrm{Ab})$ and serum complement $(\mathrm{C}$ ') in fresh serum and the corresponding Fc receptors and complement receptors on monocytes. Upon serum heat inactivation, Mtb binding is only mediated by antibodies (64). 


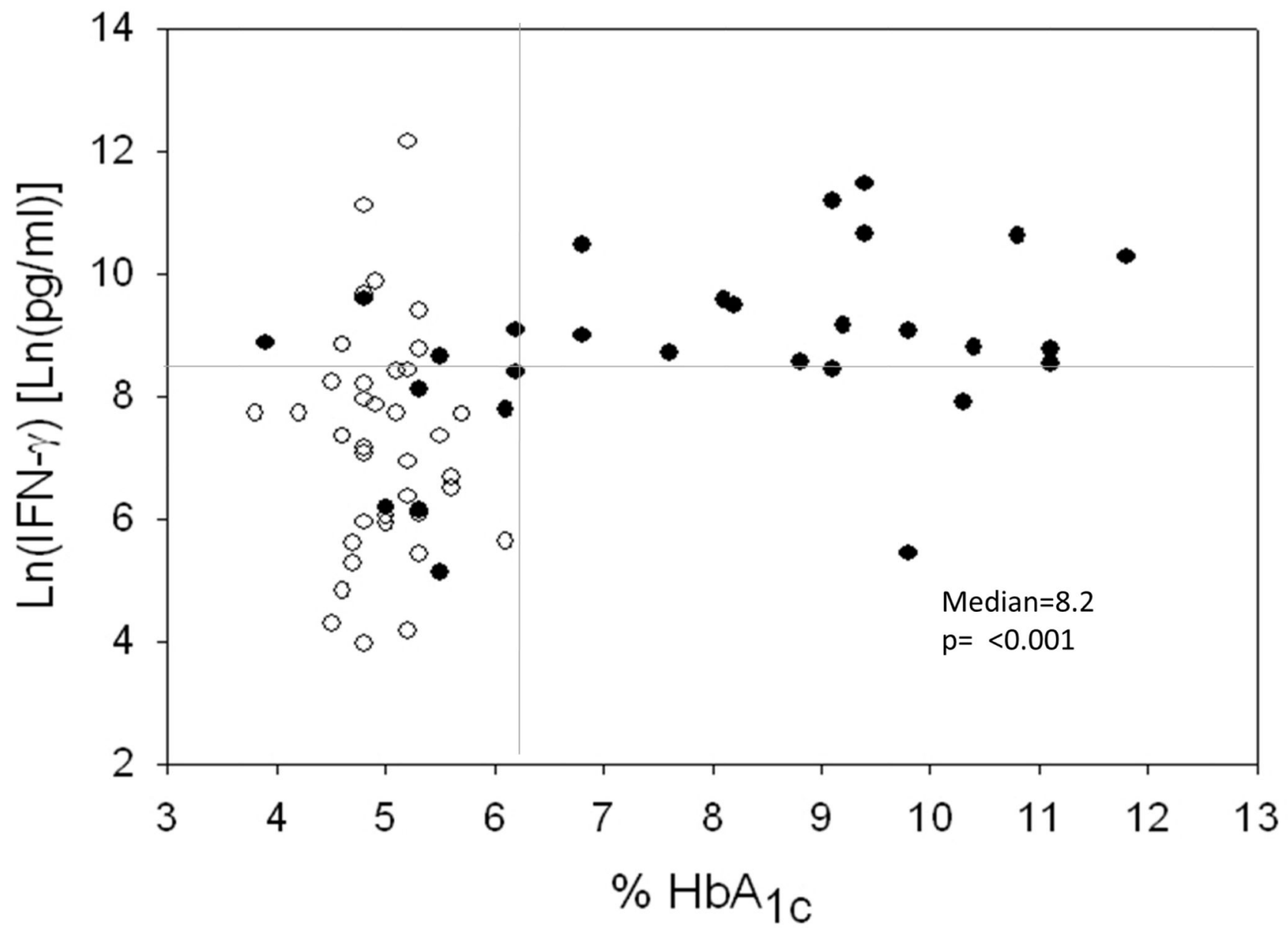

Figure 5.

Higher IFN- $\gamma$ secretion in response to PPD in TB patients with high HbA1c (versus normal HbA1c) despite their DM status. Whole blood from TB patients +/- DM was incubated with purified protein derivative (PPD) from Mtb and after 18-24h the secretion of IFN- $\gamma$ was quantified by ELISA in the culture supernatants. Results are shown in scatter plots where each circle represents one TB patient: black dots= DM and white dots= no DM. The horizontal line indicates the median IFN- $\gamma$ level. The vertical line at the HbA1c level of $6.2 \%$ of total hemoglobin indicates the upper limits of normal (left) and elevated (right) $\mathrm{HbA} 1 \mathrm{c}$ level. IFN- $\gamma$ values are provided in natural log (Ln) scale (35). 